\title{
Multimorbidity Network Surveillance: Chronic Disease Clusters and Social Disparities
}

\author{
Eun Kyong Shin ${ }^{1}$, Youngsang Kwon ${ }^{2}$, Arash Shaban-Nejad ${ }^{1}$ \\ ${ }^{1}$ Pediatrics, UTHSC, Memphis, Tennessee, United States, ${ }^{2}$ University of Memphis, Memphis, Tennessee, United States \\ Objective
}

We study how multimorbidity prevalence is related to socio-economic conditions in Memphis, TN. In addition, we demonstrate that the accumulation of chronic conditions, which is measured by affinity in multimorbidity, is unevenly distributed throughout thecity. Our research shows that not only are socio- economic disadvantages linked to a higher prevalence in each major chronic condition, but also major chronic conditions are heavily clustered in socially disadvantaged neighborhoods.

\section{Introduction}

Chronic diseases impose heavy burdens onhealth systems, economies, andsocieties [1]. Half of all Americans live with at least one of the chronic conditions and more than $75 \%$ of health care cost is associated with people with chronic diseases [2]. Multimorbidity, the coexistence of two ormore chronic conditions in an individual or a population, often require complex and ongoing care and a deep understanding of different risk factors, and their indicators.Multimorbidity has been increased over the past years and the trend is expected to continue across the U.S. Knowing how different chronic conditions are related to one another andwhat are the underlying socioeconomic factorsis crucial to design and implement effective health interventions. We introduce "multimorbidity network affinity", which measures the degree of how multiple chronic conditions are clustered within a geographic unit. Accurate estimations of how chronic conditions are spatially clustered and linked to other sociomarkers [3] and socio-economic disadvantages facilitate designing effective interventions.

\section{Methods}

Multiple datasets including major chronic condition data from the Center for Disease Control and Prevention (CDC) 500 cities, and socio-demographic data from the U.S. Census Bureau and the Environmental Systems Research Institute (ESRI) demographics data have been consistently integrated. Then, network analytics have been performed to examine the inter-relations among a selected number of major chronic conditions and their manifestations in Memphis. To checkwhether a distinctive geographic pattern in multimorbidity is present, we carried out a test using global Moran's I and Getis-Ord Gi*statistics. If apattern is detected, we use robust regression to explore how affinity isassociatedwiththe socio-economic disadvantages of the area.

\section{Results}

The network analysis confirms the existence of close relationships between various chronic conditions. Ourspatial analysisshowthat the geo-distinctive patterns of clustered comorbidities are associated with socio-economic deprivation. Statistical results suggest that neighborhoodswith higherrates of crime, poverty, and unemployment are associated with an increased likelihood of having dense clusters of chronic conditions.

\section{Conclusions}

This study shows the importance of geospatialfactors in multimorbidity network surveillance. Moreover, it demonstrates how socioeconomic disadvantages and multimorbidity network are connected. The healthdisadvantages are disproportionately accumulated in socially disadvantaged areas. Network analysis enables us to discover the links between commonly co-observed chronic diseases and explore the complexity of their interactions. This will improve the surveillance practice and facilitate timely response as well as public health planning and decision making.

\section{References}

1. Wu S-Y, Green A. The Growing Crisis of Chronic Disease in the United States. RAND Corporation. 2000. 
2. Anderson G, Horvath J. 2004. The growing burden of chronic disease in America. Public Health Rep. 119(3), 263-70. PubMed https://doi.org/10.1016/j.phr.2004.04.005

3. Shin EK, Mahajan RM, Akbilgic OA, Shaban-Nejad A. Sociomarkers and Biomarkers: Predictive Modeling in Identifying Pediatric Asthma Patients at Risk of Hospital Revisits. npj Digital Medicine (2018) 1:50; doi:10.1038/s41746-018-0056-y. 